\title{
Conciencia emocional en situaciones motrices cooperativas lúdicas y expresivas en Bachillerato: perspectiva de género
}

Emotional awareness in motor cooperative situations in ludic and expressive contexts in Non-Compulsory Secundary Education: a gender prespective

\author{
José Ignacio Alonso Roque*1 \\ jialonso@um.es \\ Marta Marín Guillén* \\ marta.marin1@um.es \\ JuAn LUIS Yuste Lucas* \\ jlyuste@um.es \\ Pere Lavega Burgués** \\ plavega@inefc.es** \\ Gemma Gea García*** \\ gmgea@ucam.edu*** \\ * Universidad de Murcia, España \\ **INEF-C Lérida, España \\ ***Universidad Católica San Antonio de Murcia, España
}

\section{Resumen:}

El propósito del presente estudio fue analizar las emociones suscitadas en situaciones motrices de cooperación lúdicas y expresivas en clase de Educación física. Para ello se administró el Cuestionario "Games and Emotions Scale" (GES) al término de cada sesión a 24 estudiantes de primero

\begin{abstract}
:
The aim of this study was to investigate the emotions experienced by eleventh grade students in physical education classes involving motor cooperative situations in ludic and expressive contexts. Data were obtained through the administration with 24 high school students of the question-
\end{abstract}

1 Dirección para correspondencia (correspondence address):

José Ignacio Alonso Roque. Departamento de Didácticas de la Expresión Plástica, Musical y Dinámica. Facultad de Educación. Universidad de Murcia. Campus de Espinardo, s/n. 30100 Espinardo (España). 
de Bachillerato. El estudio fue descriptivo, correlacional y comparativo, con dos condiciones en las que se tomaban los datos, las cuales eran los dos tipos de sesiones de la unidad didáctica realizada. Los resultados indican que independientemente del género y del tipo de sesión, se valoran como más intensas las emociones positivas, después las ambiguas y por último las negativas.

\section{Palabras clave:}

Emoción; inteligencia emocional; educación física; cooperación; expresión corporal. naire "Games and Emotions Scale" (GES) at the end of each session. The study was descriptive, correlational and comparative and included two conditions (the two different types of teaching sessions). Results indicate that, regardless of gender and type of session, positive emotions are held to be the most intense, followed by ambiguous and negative emotions.

\section{Key words:}

Emotion; emotional intelligence; physical education; cooperation; corporal expression.

\section{Résumé:}

Le but de cette étude a été d'analyser les émotions suscitées lors de situations motrices de coopération ludiques et expressives en clase d'éducation physique. Pour cela, le questionnaire "Games and Emotions Scale" (GES) a été réalisé à la fin de chaque session par 24 élèves de Primero de bachillerato. L'étude a été descriptive, corrélative et comparative, avec deux conditions où les données ont été prises, à savoir les deux types de sessions de l'unité didactique réalisées. Les résultats indiquent qu'indépendamment du genre et du type de session, les émotions positives ont été estimées plus intenses, suivies des ambigües et enfin des négatives.

\section{Mots clés:}

Émotion; intelligence émotionnelle; éducation physique; coopération; expression corporelle.

Fecha de recepción: 20-4-2016

Fecha de aceptación: 18-9-2017

\section{Introducción}

Las emociones juegan un papel determinante en las relaciones humanas desde que nacemos. Bisquerra (2000) indica que la emoción "es un estado complejo del organismo que conlleva a una excitación o perturbación que predispone a una respuesta organizada" (p. 46).

Tradicionalmente su estudio ha sido marginal hasta la llegada de los años 90 del siglo pasado cuando se inicia la denominada revolución emocional (Roselló, 1996). Las investigaciones aportadas desde diferentes disciplinas (neurociencia, biología, psicología, sociología, antropología, praxiología motriz, sistémica, etc.) constatan el papel tan relevante de las emociones en el bienestar personal y social. El carácter multidi- 
mensional de los procesos emocionales (Bisquerra, 2000; Lazarus 1991; Lewis y Havilland, 1993; Oatley y Jenkins, 1996; Parlebas, 2001) precisa estudios desde diferentes áreas de conocimiento.

La sensibilización por las emociones también ha llegado al sector educativo que se han ido interesando por la inteligencia emocional (Goleman, 1995) y el desarrollo de competencias emocionales (Dueñas, 2002; Shapiro, 1997). La educación emocional permite que el alumno aprenda el uso inteligente de sus emociones ante cualquier acontecimiento favorable o desfavorable. Para ello es necesario iniciarse en la toma de conciencia emocional y posteriormente aprender a regular las emociones negativas en situaciones adversas (Bisquerra, 2003; Mayer y Salovey, 1997).

El ámbito escolar en general y las clases de Educación Física (en adelante EF) en particular pueden ser un contexto excelente para educar las diversas áreas de la personalidad del alumno, como la afectividad. Esta disciplina se sirve de aprendizajes procedimentales en los que se aprende a dosificar su esfuerzo físico, a tomar decisiones, a expresar y manejar su afectividad y a interaccionar con el resto de compañeros, a través de la acción motriz (Lavega, Costes y Prat, 2015). Cualquier estudiante, por tanto, al saltar, correr o pasar un balón, activa de manera unitaria las diferentes dimensiones de su personalidad (biológica, cognitiva, emocional y social) (Lagardera y Lavega, 2003), a través de su conducta motriz. Este concepto integrador remite a la "organización significativa del comportamiento motor, es decir, es el comportamiento motor en cuanto portador de significado" (Parlebas, 2001, p. 85). Al participar un alumno en una clase de EF, da testimonio de su biografía motriz y de su bienestar personal, social y afectivo. Por ello la EF moderna debería convertirse en una auténtica pedagogía de conductas motrices (Lavega, 2005), en las que el crecimiento emocional debería ocupar un lugar destacado (Alonso, Lavega y Reche, 2011; Arruza, 2002; Lavega, Alonso, Araujo, et al., 2011; Lavega, Filella, Agulló, Soldevila y March, 2011; Liukkonen, Barkoukis, Watt y Jaakkola, 2010; Salmerón, 2002).

Igual que ocurre en otros escenarios vitales, cuando un profesor invita a un estudiante a realizar un juego, una tarea o un deporte le está introduciendo en un sistema social. La persona se ve inmersa en continuos sistemas sociales, a menudo asociados mayoritariamente al género masculino o femenino (Gea, Alonso, Rodríguez y Caballero, 2017). Las relaciones que se establecen entre ambos géneros se aprenden social- 
mente y además pueden ir cambiando en el tiempo. En el proceso de socialización cada persona va configurando un estereotipo social en valores, comportamientos y actitudes.

En EF el uso acentuado hacia el deporte ha originado un escenario que refleja los estereotipos masculino que han favorecido sistemas sociales con predominio de este rol (Mosquera y Puig, 2002). La incorporación progresiva de las mujeres al deporte ha diversificado la forma de entender estas actividades (Knoppers y Elling, 2001; Soler, 2009). Las mujeres han sido asociadas con estereotipos cercanos a inestabilidad emocional, la dulzura, la ternura, la comprensión o la cooperación, mientras que a los hombres se les ha asociado con el control emocional, el dinamismo, la agresividad, la competitividad o el liderazgo. Actualmente es necesario estudiar cómo se está redefiniendo la masculinidad en el deporte y el papel que pueden desempeñar las emociones en este proceso.

Teniendo en cuenta estos cambios, se podría pensar que, en una sesión de EF, los chicos y las chicas pueden vivir emociones distintas en función de si se les pide participar en situaciones motrices deportivas asociadas a uno de los dos géneros (Gea et al., 2017), como el rugby o el judo, determinados juegos tradicionales como saltar a la cuerda, danzar o expresar con su cuerpo.

Según autores como Bisquerra (2000) y Lazarus (1991b) ante cualquier estímulo, como una situación motriz en EF, la persona valora la situación según sus expectativas, de manera que si ese estímulo le aporta bienestar, se desencadenarán emociones positivas como alegría, humor, amor o felicidad. En cambio, si le provoca malestar, se activarán emociones negativas como tristeza, miedo, rechazo, vergüenza, ira o ansiedad. Finalmente, se encuentran las emociones ambiguas o neutras, como sorpresa, esperanza o compasión ya que según las circunstancias pueden tener una orientación hacia las emociones positivas o negativas.

Si los chicos perciben una situación motriz como "algo" de chicas, es fácil que desencadenen emociones negativas y lo mismo en sentido contrario respecto a un juego considerado de chicos si se le presentara a una alumna. Igualmente, podemos pensar que las emociones van a ser diferentes si se pide competir a los alumnos y determinados estudiantes tienden a ganar o perder siempre. De ahí la necesidad de generar conocimientos que permitan desvelar la correspondencia entre la naturaleza de las prácticas motrices y los efectos que generan en la vivencia emocional de sus participantes (e.g., Alonso, Lavega y Reche, 2011; Lave- 
ga, Alonso, Araujo, et al., 2011; Lavega, Alonso, Etxebeste, Lagardera y March, 2014; Lavega et al., 2011).

Desde esta perspectiva, estudios sobre género han mostrado que hombres y mujeres no siempre se comportan igual emocionalmente. Las mujeres serían más propensas a expresar emociones que los hombres (Brody y Hall, 2000; Fabes y Martín, 1991). Esta circunstancia puede originar situaciones de desequilibrio o desigualdad en la educación emocional (Sánchez, Fernández-Berrocal, Montañés y Latorre, 2008). En el ámbito de la EF, se han encontrado diferencias sustanciales entre ambos géneros en alumnos universitarios ante situaciones de juego con competición, donde las mujeres expresaron menor intensidad respecto a emociones negativas ante la derrota (Gea et al., 2017; Lavega et al., 2011).

La aparente dispersión que generan los centenares de situaciones motrices de las que se puede servir el profesor de EF, se pueden concretar en familias de prácticas atendiendo al sistema de relaciones motrices que originan. Según el tipo de interacción motriz, se identifican cuatro grandes dominios o familias (Parlebas, 2001): a) psicomotor: en el que los participantes intervienen sin interactuar con otros jugadores; b) cooperación: en el que al menos dos alumnos interactúan positivamente para alcanzar un objetivo común; c) oposición: en el que dos o más jugadores se oponen para superar a los rivales; d) cooperación-oposición: en el que se comparten las relaciones de ayuda y hostilidad motriz. Cada uno de estos dominios traslada a los alumnos a vivir experiencias motrices distintas que a su vez van a poder originar efectos diversos en sus conductas motrices (Lagardera y Lavega 2003; Parlebas, 2001). En este artículo la atención se dirige hacia el dominio cooperativo, en el que se pueden encontrar dos tipos de prácticas: los juegos cooperativos y las situaciones motrices de expresión. Estas prácticas se han considerado por la presencia en el currículum educativo de la etapa y por haber sido estudiadas en ámbitos universitarios de forma separada (Lavega Mateu, Lagardera y Filella, 2010; Lavega, Filella, Lagardera, Mateu y Ochoa, 2013).

Los juegos cooperativos pueden ser un excelente medio para activar procesos como el control del propio cuerpo, automatismos-estereotipos motores, repetición programada, toma de decisiones, uso de capacidades reflexivas o cognitivas, comunicación motriz con los demás (búsqueda de objetivos comunes) y pacto de reglas con los otros (Parlebas, 2001; Lavega y Lagardera, 2003). Los procesos activados en el alumno, 
junto con las actitudes a las que van ligados, pueden provocar diferentes intensidades emocionales en el alumno. Estudios previos (Lavega et al., 2011) compararon los resultados en los diferentes dominios de acción motriz, mostrando que los juegos cooperativos activaron las emociones positivas más intensas entre los estudiantes universitarios.

Por otro lado, las situaciones motrices expresivas se encuentran integradas en las diferentes etapas educativas y permiten trabajar la comunicación expresiva entre los alumnos, así como otros contenidos como danza, ritmo o autoexpresión a través de la motricidad. Al participar en estas tareas, los protagonistas deben realizar conductas motrices Comunicativas, creativas y artísticas/estéticas (Ramos, Cuéllar y Jiménez., 2009), en las que la comunicación es de carácter referencial y poético (Mateu, 2010; Torrents, Mateu, Planas, y Dinusôva, 2011). Según Izquierdo (2000) las emociones tienen un papel regulador de los contactos, de las relaciones y en la comprensión de los mensajes. Entendemos que dramatizar, llevar un ritmo, el contacto corporal, la comunicación no verbal, etc., son también medios potenciales para suscitar y regular emociones, ya que en su propia naturaleza está la transmisión de un mensaje emotivo o afectivo, lo que lo hace susceptible de ser investigado.

Teniendo en cuenta el interés que suscita conocer cómo se producen las vivencias de los alumnos durante el proceso de enseñanza-aprendizaje de ambos tipos de situaciones motrices, el objetivo del estudio fue analizar la intensidad de emociones positivas, negativas y ambiguas que experimentaron las alumnas y los alumnos de bachillerato al participar en una unidad didáctica basada en la cooperación, mediante situaciones motrices cooperativas lúdicas y expresivas.

\section{Método}

\section{Participantes}

En el presente estudio participaron 24 alumnos (14 chicos y 10 chicas; rango de edad de 16-17 años) de $1^{\circ}$ de Bachillerato de un IES de la Región de Murcia. Se consiguieron todos los consentimientos informados de los padres de los alumnos, así como del centro educativo en cuestión. Cada alumno generó un total de 135 valoraciones de intensidades emocionales durante toda la intervención. 


\section{Instrumentos y procedimientos}

El diseño del estudio responde a un trabajo descriptivo, correlacional y comparativo, con dos condiciones. Se diseñó y aplicó una unidad didáctica de ocho sesiones dividida en cuatro sesiones (45 minutos cada una) con situaciones motrices expresivas y cuatro con situaciones motrices de juegos cooperativos que conformaron la variable independiente del estudio. La unidad didáctica planteada se presentó bajo una estructura metodológica cooperativa, en la que se buscaba el trabajo conjunto por pequeños grupos y situaciones de resolución de reto grupal. Se aplicaron estrategias globales en la práctica y se potenciaron recursos cooperativos para el desarrollo de las sesiones, como plantillas de mejora de grupo. A su vez se potenció una participación máxima en la resolución de las tareas por parte del alumno, cercano a un estilo cooperativo y socializador.

En un primer lugar se explicó a los participantes el objetivo básico de las sesiones, ya que se les pediría que se evaluasen a nivel emocional. La duración total de la intervención fue de cuatro semanas. Las actividades de enseñanza-aprendizaje fueron revisadas por el profesor habitualdel grupo participante y por dos expertos con más de diez años de experiencia en juegos motores y expresión corporal, analizando la lógica interna de todas las situaciones motrices que componían cada una de las sesiones de los dos tipos de unidad didáctica. Se aplicaron primero las sesiones relativas a situaciones motrices expresivas y una vez terminadas se realizaron las sesiones de juegos cooperativos.

Para el registro de los datos se administró la escala Games and Emotions Scale (GES) validada por Lavega, March y Filella (2013) para identificar de cero a diez la intensidad de trece emociones básicas experimentadas durante la sesión, lo que ha sido considerara como la variable dependiente del presente estudio. Las emociones a valorar fueron: alegría, humor, amor y felicidad (emociones positivas), tristeza, miedo, ansiedad, ira, rechazo y vergüenza (emociones negativas); compasión, sorpresa y esperanza (emociones ambiguas).

Las principales propiedades psicométricas de la escala GES utilizada en el estudio fueron: a) aceptabilidad: los valores de asimetría $(<2.0)$ y curtosis $(<7.0)$ cumplieron los criterios de normalidad; b) fiabilidad: para evaluar la consistencia interna en toda la escala se calculó el coeficiente alpha de Cronbach $(\mathrm{n}=851 ; \alpha=.92)$. Se obtuvieron valores similares para cada tipo de emoción: positiva $(\alpha=.92)$, negativa $(\alpha=.88)$ 
y ambigua $(\alpha=.93)$; c) el análisis factorial confirmatorio reprodujo de manera adecuada la estructura de la escala y mostró índices aceptables $(\mathrm{CMIN} / \mathrm{g} . \mathrm{I} .=7.014 ; \mathrm{NFI}=.813 ; \mathrm{IFI}=.836 ; \mathrm{CFI}=.833 ; \mathrm{RMSEA}=.08$ $(\mathrm{LO} 90=.072-\mathrm{HI90}=.09)$.

Previo al registro de datos se realizó una sesión de formación en emociones que sirvió también como familiarización con el instrumento y con la presencia de los investigadores durante las sesiones. La formación incluía situaciones motrices diversas, expresivas y lúdicas con diferentes relaciones motrices, en los cuáles los alumnos tenían que representar varias emociones a los demás compañeros, con el fin de vivenciar las emociones explicadas.

\section{Análisis estadístico}

Las variables independientes del estudio fueron intensidad emocional de las trece emociones descritas por el modelo de Bisquerra (2000), mientras que el tipo de sesión utilizado (sesión de juego motor o sesión de expresión corporal) fue la variable independiente. Para todos los análisis se tuvo en cuenta el género de los participantes. Los datos fueron tratados mediante estadística descriptiva, mientras que, para comparar la respuesta de las diferentes emociones en cada una de las sesiones, se aplicó el modelo lineal general de medidas repetidas y, en el supuesto de hallar diferencias significativas, se procedió a las comparaciones múltiples post-hoc mediante el ajuste de Bonferroni.

Para comparar la relación existente entre el tipo de emoción de los participantes en las diferentes sesiones analizadas en función del género y del tipo de sesión realizada, se aplicó la T-Student para muestras independientes. El estudio de la regresión logística permitió calcular el efecto del género y del tipo de sesión sobre las emociones. Para establecer la significación estadística, se utilizó el valor de $p<.05$.

\section{Resultados y discusión}

El objetivo del estudio fue analizar la intensidad de emociones positivas, negativas y ambiguas que experimentó el alumnado de bachillerato al participar en una unidad didáctica basada en la cooperación, mediante juegos cooperativos y situaciones motrices de expresión. 
En la Tabla 1 se exponen las medias obtenidas para cada una de las emociones. Antes de pasar a analizar los datos, se comprobaron las diferencias estadísticas entre cada una de las emociones sin tener en cuenta otros factores. Para este fin se aplicó el modelo lineal general, con el cual pretendemos confirmar la potencia de la significación de los datos obtenidos en la Tabla $1(p<.001)$.

Tabla 1

Distribución de intensidades medias de las emociones suscitadas

\begin{tabular}{clcccc}
\hline \multirow{2}{*}{ Tipo Emoción } & Emociones & N & Media & $\begin{array}{c}\text { Desviación } \\
\text { Típica }\end{array}$ & $\begin{array}{c}\text { Error típ. } \\
\text { de la media }\end{array}$ \\
\hline \multirow{4}{*}{$\begin{array}{c}\text { Emociones Positivas } \\
(+)\end{array}$} & Felicidad (+) & 135 & 7,39 & 1,693 &, 146 \\
& Alegría (+) & 135 & 7,38 & 1,688 &, 145 \\
& Humor (+) & 135 & 7,19 & 2,581 &, 222 \\
& Amor (+) & 135 & 5,41 & 2,487 &, 214 \\
\hline \multirow{5}{*}{ Emociones Negativas } \\
(-) & Ira (-) & 135 & 1,63 & 2,285 &, 197 \\
& Rechazo (-) & 135 & 1,45 & 2,154 &, 185 \\
& Mristeza (-) & 135 & 1,84 & 2,166 &, 186 \\
& Aiedo (-) & 135 & 1,67 & 2,565 &, 221 \\
Emocionedad (-) & 135 & 2,40 & 2,765 &, 222 \\
(a) & Vergüenza (-) & 135 & 1,91 & 2,297 &, 198 \\
\hline \multirow{2}{*}{ (ambiguas } & Compasión (a) & 135 & 4,25 & 2,527 &, 217 \\
& Sorpresa (a) & 135 & 5,59 & 2,887 &, 248 \\
& Esperanza (a) & 135 & 5,36 & 3,075 &, 265 \\
\hline
\end{tabular}

Nota. Basadas en las medias marginales estimadas. La diferencia de las medias es significativa al nivel ,05. Ajuste para comparaciones múltiples: Bonferroni.

No se encontraron diferencias significativas al comparar emociones positivas entre sí, para felicidad (7.39) y humor (7.19). De la misma forma no hubo diferencias significativas en el caso de las emociones negativas comparadas entre sí (ira, 2.28; rechazo, 2.15; tristeza, 2.66). Cuando la comparación se realizó entre una emoción positiva y otra negativa, se encontraron diferencias significativas para la felicidad (7.39) y tristeza (1.84) $(p<.001)$.

En la Tabla 2 se exponen los resultados obtenidos a través del modelo lineal general. Para facilitar el análisis de los datos por pares, se expone la comparación de una emoción positiva (felicidad) con el resto de emociones. La selección de esta emoción para ser comparada con el resto vino determinada por su alusión muy significativa en la 
literatura revisada, además de por ser la emoción que obtuvieron intensidades más elevadas (felicidad en el grupo de emociones positivas). La felicidad se encuentra relacionada con la aparición del bienestar y estado positivo (Bisquerra, 2000; Goleman, 1995; Díaz, RodríguezCarvajal, Blanco, Moreno-Jiménez, Gallardo, Valle y Van Dierendonck, 2006), lo que nos permite comparar estadísticamente con el resto de las emociones analizadas en el estudio y por lo tanto poder contrastar la vivencia emocional de todo el proceso educativo estudiado.

Tabla 2

Modelo lineal general. Comparación individualizada de Felicidad y Ansiedad con el resto

\begin{tabular}{|c|c|c|c|c|}
\hline \multirow[t]{2}{*}{ (I) factor 1} & \multicolumn{3}{|c|}{ Diferencia medias } & $\begin{array}{c}\text { Significación } \\
p \\
\end{array}$ \\
\hline & & Límite inferior & Límite superior & \\
\hline Felicidad & Alegría & 0.015 & .127 & 1.000 \\
\hline & Humor & 0.200 & 241 & 1.000 \\
\hline & Amor & $1.978\left(^{*}\right)$ & .220 & $<.001$ \\
\hline & Ira & $5.763\left(^{*}\right)$ & 290 & $<.001$ \\
\hline & Rechazo & $5.941(*)$ & .274 & $<.001$ \\
\hline & Tristeza & $5.556\left(^{*}\right)$ & .278 & $<.001$ \\
\hline & Miedo & $5.719\left(^{*}\right)$ & .295 & $<.001$ \\
\hline & Ansiedad & $4.993(*)$ & 311 & $<.001$ \\
\hline & Vergüenza & $5.481(*)$ & .259 & $<.001$ \\
\hline & Compasión & $3.141\left(^{*}\right)$ & .271 & $<.001$ \\
\hline & Sorpresa & $1.800(*)$ & 279 & $<.001$ \\
\hline & Esperanza & $\left.2.030{ }^{*}\right)$ & .307 & $<.001$ \\
\hline
\end{tabular}

Nota: Basadas en las medias marginales estimadas. ${ }^{*}$ La diferencia de las medias fue significativa $p<, 05$. Ajuste para comparaciones múltiples: Bonferroni.

Como podemos observar en la Tabla 2, se observaron diferencias significativas $(p<.001)$ entre la emoción positiva de felicidad y las emociones negativas de tristeza, miedo, ansiedad, rechazo y vergüenza (emociones negativas). También existieron diferencias significativas $(p<.001)$ con las emociones ambiguas de compasión, sorpresa y esperanza. Igualmente se observaron diferencias significativas $(p<.001)$ con la emoción positiva de amor, que son emociones negativas, excepto el amor y la esperanza. En todos esos casos la felicidad registró valores superiores $(M=7.39)$ respecto 
a esas diez emociones. No obstante no se encontraron diferencias significativas entre la felicidad y las emociones positivas de alegría y humor $(p=1)$.

Las emociones marcadas por los alumnos como más intensas en todas las sesiones son las positivas, destacando entre las mismas la felicidad, alegría y humor. Estos datos apoyarían otros estudios revisados (Liukkonen et al., 2010) que sostienen que el juego y el clima adecuado promueven el aprendizaje de una manera agradable y placentera.

Las emociones negativas adoptaron unos valores muy bajos con respecto a las positivas, siendo la más alta la ansiedad que no superó la media (5). Este dato puede deberse a la coincidencia del periodo de exámenes en el momento de aplicación de la UD. Esto implicaría tener en cuenta la limitación del momento académico de los alumnos en el momento de la experiencia como un factor presente para elegir un tipo de sesiones u otro. Sin duda sería un aspecto a profundizar en futuros estudios que se orienten a elementos de la lógica externa como parte de la vivencia emocional. Las bajas puntuaciones en emociones negativas coinciden con resultados encontrados en estudios similares (Alonso, Lavega y Reche, 2011; Lavega, Alonso, Araujo, et al., 2011; Lavega et al., 2011), denotando que las situaciones motrices lúdicas y expresivas van asociadas a una vivencia positiva. En nuestro estudio las emociones más valoradas fueron felicidad, alegría y humor. Las menos puntuadas fueron el rechazo y la ira. Esto puede indicar que independientemente del tipo de situación motriz, los participantes valoraron con puntaciones más altas las emociones positivas y con puntuaciones más bajas las emociones negativas. La EF, sería una excelente herramienta para el trabajo en emociones que generen bienestar con la situación planteada en las clases. Este dato nos indica que debemos seguir profundizando en otros contenidos y dominios de acción motriz, además de apuntar que el ritmo y clima de la clase, así como las relaciones alumno-alumno y profesor-alumno, pueden favorecer el sentimiento de emociones positivas en el proceso. Finalmente encontramos que, para las emociones ambiguas, la tendencia es a acercarse a lo positivo de la vivencia. Esto reforzaría que la experiencia fue marcada para los alumnos participantes como algo agradable y que produjo bienestar, aunque debe profundizarse el conocimiento de este tipo de emociones en EF.

Las clases de EF de juegos cooperativos y expresión corporal, suscitaron en los alumnos participantes conductas prosociales, jugando las emociones un papel importante (Eldar, 2008; Lavega et al., 2010). Los 
resultados mostraron que las emociones positivas suscitadas en nuestro estudio son intensas en ambos tipos de sesiones. Desde esta óptica, Lavega et al. (2011) consideran que los juegos cooperativos, sobre todo los no competitivos, son los más apropiados para activar emociones positivas intensas. Los resultados sugieren que el tipo de juegos a elegir es lo primero que el docente debe estudiar para educar en emociones.

En cuanto a la relación existente entre la emoción suscitada en función del género del alumno, destaca únicamente la significación estadística encontrada para el amor $(p=.008)$. Los datos no mostraron diferencias significativas respecto a las demás emociones analizadas y el género de los alumnos. Respecto al "amor", la media de los chicos fue de 4,98 mientras que la de las mujeres fue de $6.14(p<.05)$. Este resultado nos lleva a pensar que las chicas de nuestro estudio, experimentaron esta emoción más intensamente que los chicos. Estos datos sugieren una mayor profundización en el motivo de esta diferenciación, teniendo en cuenta que no se da en el resto de emociones positivas. En general, podríamos decir que tanto chicos como chicas experimentaron las emociones positivas con igual intensidad, resultados similares a estudios en otras poblaciones (Gea et al., 2017). En el estudio de Hall y Matsumoto (2004), cuyos datos indicaban que las chicas puntuaron más alto las emociones negativas que los chicos, fueron opuestos a los datos hallados en nuestro estudio, donde los chicos puntuaron más alto las emociones negativas a excepción del miedo y la ansiedad. La casi igualdad de género en nuestros datos, contrasta también con los estudios de Brody y Hall (2000) y Fabes y Martín (1991), que mostraban diferencias en las mujeres en cuanto a expresar más intensidad emocional que los hombres. Los resultados encontrados, en cambio, van en consonancia con los aportados por Lavega et al. (2011) y Gea et al. (2017), donde en los juegos realizados, los hombres y las mujeres mostraron tendencias parecidas en los distintos dominios de acción motriz y en las tres clases de emociones. Pero la mayor intensidad emocional se mostraba en la población masculina. Sin duda, la variable género parece estar influenciada por otros factores, por lo que será necesaria la realización de estudios que traten estas variables desde otras ópticas o diseños metodológicos. Sin duda el contexto educativo del estudio y la paulatina modificación de estereotipos de género (Knoppers, A. y Elling, A. 2001; Soler, 2009), irán mostrando que chicos y chicas pueden expresar emociones de forma similar ante las clases de EF. 
Abundando en los tipos de sesiones planteadas se observa en la Tabla 3 que existieron diferencias significativas respecto a las emociones analizadas y el tipo de sesión en la "alegría" ( $p=.002)$.

Tabla 3

Prueba T- Student para muestras independientes. Relación existente entre la emoción positiva suscitada y el tipo de sesión

\begin{tabular}{llccc}
\hline & TIPO JUEGO & $\mathrm{N}$ & Media & Significación \\
\hline \multirow{2}{*}{ Felicidad } & Expresión motriz & 75 & 7.16 & .074 \\
\cline { 2 - 5 } & Juego cooperativo & 60 & 7.68 & \\
\hline \multirow{2}{*}{ Alegría } & Expresión motriz & 75 & 6.97 & .002 \\
\cline { 2 - 5 } & Juego cooperativo & 60 & 7.88 & \\
\hline \multirow{2}{*}{ Humor } & Expresión motriz & 75 & 7.15 & .818 \\
\cline { 2 - 5 } & Juego cooperativo & 60 & 7.25 & \\
\hline \multirow{2}{*}{ Amor } & Expresión motriz & 75 & 5.32 & .622 \\
\cline { 2 - 5 } & Juego cooperativo & 60 & 5.53 & \\
\hline
\end{tabular}

Los valores obtenidos fueron significativos siendo los juegos cooperativos, donde se suscita en mayor medida esta emoción. Si nos centramos en el "miedo", obtuvo una media de 2.17 en expresión corporal y de 1.05 en juegos cooperativos. La "ansiedad" en juegos cooperativos no alcanzó un valor elevado, sin embargo fueron resultados significativos $(p=.004)$. Y por último la "vergüenza" en ambos tipos de sesiones tampoco superó el valor medio (5), sin embargo se obtuvo un resultado significativo $(p<.001)$. La regresión logística verificó que las diferencias halladas, en función del género y del tipo de sesión, fueron significativas.

La prueba T-Student para muestras independientes y la regresión logística marcaron que no existieron diferencias significativas respecto a las emociones analizadas y el género de los alumnos, exceptuando el "amor". Se confirmó la significación dada entre la variable independiente "amor" y la dependiente "género" $(p=.01)$. Ocurrió lo mismo con las emociones que obtuvieron diferencias significativas en función del tipo de sesión.

Estos datos nos permiten afirmar que para los alumnos participantes, los juegos cooperativos potenciaron las emociones positivas, al igual que la expresión corporal, en menor medida. La vergüenza y el miedo fueron más suscitados en expresión corporal, pero siempre dentro de las puntuaciones 
más bajas obtenidas en las emociones negativas suscitadas. Los resultados van en la dirección aportada por otros estudios (Alonso, Etxebeste y Lavega, 2010; Alonso, Lavega y Reche, 2011; Lavega et al., 2011), en el sentido de entender los juegos cooperativos como una situación motriz idónea para suscitar emociones positivas en contextos educativos. Estos resultados confirman de igual modo que los valores prosociales pueden trabajarse en EF (Eldar, 2008), dado que la conducta cooperativa, asociada a la vivencia positiva de emociones, puede ser una unión importante en las sesiones.

\section{Conclusiones}

Tras el análisis de los resultados obtenidos podemos concluir lo siguiente:

1. Las emociones positivas fueron las más intensas, mientras que las negativas se valoraron más bajas. Las emociones más intensas fueron la felicidad, la alegría y el humor, mientras que las menos intensas fueron el rechazo y la ira.

2. Los juegos cooperativos potenciaron las emociones positivas, al igual que la expresión corporal, pero esta en menor medida.

3. Tanto los chicos como las chicas, experimentaron la misma intensidad emocional positiva.

\section{Aplicaciones prácticas y líneas de investigación futuras}

Es necesario que el alumno sepa reconocer un tipo de emoción, afrontarla y gestionarla, tanto para sí mismo, como para los demás. La EF puede ser un excelente medio para el trabajo de la educación emocional, ya que, a través de diferentes situaciones motrices los alumnos son capaces de vivenciar gran cantidad de emociones. La conducta motriz, como elemento integrador de la persona, que permite actuar desde todos sus aspectos, se muestra como un marco pertinente para entender la importancia de la persona en movimiento, expresivo o cooperativo. El hecho de no encontrar aspectos diferenciadores de género puede abrir una puerta a un cambio dentro de los patrones de género. Es posible que tengamos delante un marco de actuación donde los género parten con las mismas posibilidades de aprendizaje, dado que las vivencias son similares y no excluyentes. 
José Ignacio Alonso Roque, Marta Marín Guillén, Juan luis Yuste lucas, Pere Lavega

Como futuras necesidades de investigación es necesario 1) profundizar en los factores que pueden influir en las emociones suscitadas en los alumnos, para que los docentes puedan tener en cuenta aquellos que contribuyen al sentimiento de emociones positivas y regular aquellos otros que contribuyen al sentimiento de emociones negativas; 2) analizar si para una adecuada madurez emocional sería conveniente que los alumnos también vivencien emociones negativas aunque de forma controlada para sentirlas en menor grado; 3) profundizar en el motivo de las diferencias existentes que contribuyen al sentimiento del "amor" en mayor grado en las chicas y determinar si realmente se está dando un cambio en la expresión de emociones en función del género, o bien si es el contexto educativo el que haya hecho que no se dieran diferencias; 4) buscar en la figura del docente un generador de vivencias positivas a través de sus conductas docentes.

\section{Agradecimientos}

Parte de este trabajo ha recibido financiación del Ministerio de Ciencia e Innovación. Proyectos I+D+i. Ref. DEP2010-21626-C03-01; DEP201021626-C03-02; DEP2010-21626-C03-03. También ha recibido financiación de la Agencia de Gestió d'Ajuts Universitaris i de Recerca de la Generalitat (AGAUR-INEFC). Ref. 2009SGR1404; VCP/3346/2009.

\section{Referencias}

Alonso, J. I., Etxebeste, J. y Lavega, P. (2010). Análisis de emociones suscitadas en juegos motores con y sin competición. En VI Congreso Internacional de la Asociación Española de Ciencias del Deporte, Elche: Universidad Miguel Hernández.

Alonso, J.I., Lavega, P. y Reche, F. (2011). Análisis de la vivencia emocional desde la perspectiva de género en juegos deportivos de cooperación con oposición en alumnos de Bachillerato. En Actas Congreso Internacional en Ciencias de la Actividad Física y del Deporte: Investigación, desarrollo e innovación. Vitoria: UPV.

Arruza, J. (2002). Las emociones orientadas hacia la acción en el contexto de la actividad física y el deporte. Tándem, 7, 56-74.

Biquerra, R. (2000). Educación emocional y bienestar. Barcelona: Praxis

Bisquerra, R. (2003). Educación emocional y competencias básicas para la vida. Revista de Investigación Educativa, 21(1), 7-43.

Brody, L. R. y Hall, J. A. (2000). Gender, emotion and expresion. In M. Lewis y J.M. 
Conciencia emocional en situaciones motrices cooperativas lúdicas y expresivas en Bachillerato: perspectiva de género

José Ignacio Alonso Roque, Marta Marín Guillén, Juan luis Yuste Lucas, Pere Lavega Burgués y Gemma Gea García

Haviland-Jones (Eds.). Handbook of emotions (pp. 338- 349). New York: Guilford Press.

Díaz, D., Rodríguez-Carvajal, R., Blanco, A., Moreno-Jiménez, B., Gallardo, I., Valle, C. y Van Dierendonck, D. (2006). Adaptación española de las escalas de bienestar psicológico de RYFF. Psicothema, 18(3), 572-577.

Dueñas, M. (2002). Importancia de la inteligencia emocional. Un nuevo reto para la orientación educativa. Revista Educación XX1, 5, 77-96.

Eldar, E. (2008). Education \& Treatment of children. Education; Family and marriage. Social sciences, 32(3), 435-457.

Fabes, R. y Martin, C. (1991). Gender and age stereotypes of emotionality. Personality and Social Psychology Bulletin, 17, 532-540.

Gea, G., Alonso, J., Rodríguez, J. y Caballero, M. (2017). ¿Es la vivencia emocional cuestión de género? Análisis de juegos motores de oposición en universitarios. Revista de Investigación Educativa, 35(1), 269-283.

Goleman, D. (1995). Inteligencia emocional. Barcelona: Kairós.

Hall, J. y Matsumoto, D. (2004). Gender Differences in Judgments of Multiple Emotions From Facial Expressions. Emotion, 4(2), 201-206.

Knoppers, A. y Elling, A. (2001). Organizing masculinities and feminities: the gendered sporting body. In J. Steenbergen, P. De Knopp y A. Elling (Eds.). Values and norms in sport (pp. 171-194). Aachen: Meyer \& Meyer sport.

Izquierdo, C. (2000). Comunicación interpersonal y crecimiento emocional en centros educativos: un modelo interpretativo. Educar, 26, 127-149.

Lagardera, F. y Lavega, P. (2003). Introducción a la Praxiología Motriz. Barcelona: Paidotribo.

Lavega, P. (2005). La educación física como pedagogía de las conductas motrices. Tándem: Didáctica de la educación física, 18, 79-101.

Lavega, P., Costes, A. y Prat, Q. (2015). Educar competencias emocionales en futuros profesores de educación física. Revista Interuniversitaria de Formación del Profesorado, 83(29.2), 61-74.

Lavega, P., Filella, G., Agulló, M., Soldevila, B. y March, J. (2011). Conocer las emociones a través de juegos: ayuda para los futuros docentes en la toma de decisiones. Electronic Journal of Research in Educational Psychology, 9(2), 617-640.

Lavega, P., Filella, G., Lagardera, F., Mateu, M. y Ochoa, J. (2013). Juegos motores y emociones. Cultura y educación, 25(3), 347-360.

Lavega, P., Alonso, J., Etxebeste, J., Lagardera, F. y March, J. (2014). Relationship between traditional games and the intensity of emotions experienced by participants. Research Quarterly for Exercise and Sport, 85(4), 457-467.

Lavega, P., Alonso, J., Araujo, P., Etxebeste, J., Jaqueira, A., Lagardera, F., March, J. y Rodríguez, J.P. (2011). Investigar en el aula de educación física. Perspectiva de género, Juegos deportivos, y emociones. En Actas I Congreso de Inteligencia Emocional a las Organizaciones. La Inteligencia emocional como ventaja competitiva. Lleida: GROP.

Lavega, P.; March, J.; Filella, G. (2013). Juegos deportivos y emociones. Propiedades psicométricas de la escala GES para ser aplicada en la Educación Física y el Deporte. Revista de Investigación Educativa, 31(1), 151-165. 
Conciencia emocional en situaciones motrices cooperativas lúdicas y expresivas en

Bachillerato: perspectiva de género

José Ignacio Alonso Roque, Marta Marín Guillén, Juan luis Yuste lucas, Pere Lavega Burgués y Gemma Gea García

Lavega, P., Mateu, M., Lagardera, F. y Filella, G. (2010) Educar emociones positivas a través de los juegos deportivos. En M. A. Torralba, M. De Fuentes, J. Calvo y J. F. Cardozo (Eds.), Docencia, innovación e investigación en educación física. (pp. 111-139). Barcelona: INDE Publicaciones

Lazarus, R. (1991a). Cognition and Motivation in emotion. American Psychologist, 46, 352-367.

Lazarus, R. (1991b). Emotion and adaptation. Nueva York: Oxford University Press.

Lewis, M. y Haviland, J.M. (1993). Handbook of emotions. Nueva York: Guilford.

Liukkonen, J., Barkoukis, V., Watt, A. y Jaakkola, T. (2010). Motivational Climate and Students' Emotional Experiences and Effort in Physical Education. The Journal of Educational Research, 103, 295-308. DOI:10.1080/00220670903383044.

Mateu, M. (2010). Observación y análisis de la expresión motriz escénica. Estudio de la lógica interna de los espectáculos artísticos profesionales: Cirque du Soleil (19862005). Tesis doctoral no publicada. INEFC. Barcelona: Universidad de Barcelona.

Mayer, J.D. y Salovey, P. (1997). What is emotional intelligence? In P. Salovey y D, Sluyter (Eds.), Emotional Development and Emotional Intelligence: implications for educators (pp. 3-31). New York: Basic Books.

Mosquera, M.J. y Puig, N. (2002). Género y edad en el deporte. En M. García Ferrando, F. Lagardera y N. Puig (Eds.), Sociología del Deporte (pp. 114-141). Madrid: Alianza Editorial.

Oatley, K. y Jenkins, J. (1996). Understanding emotions. Cambridge, Mass: Blackwell Publishers.

Parlebas, P. (2001). Juegos, deportes y sociedades. Léxico de praxiología motriz. Barcelona: Paidotribo.

Ramos, J., Cuéllar, M. y Jiménez, F. (2009). Nuevo retos en el desarrollo curricular de la expresión corporal. Emásf, 14,142-149.

Roselló, J. (1996). Introducción a la psicología del sentimiento: Motivación y emoción. Barcelona: Palma.

Salmerón, P. (2002). Evolución de los conceptos sobre inteligencia. Planteamientos actuales de la inteligencia emocional para la orientación educativa. Revista Educación $X X 1,5,97-122$.

Sánchez, M., Fernández-Berrocal, P., Montañés, J. y Latorre, J. (2008). ¿Es la inteligencia emocional una cuestión de género? Socialización de las competencias emocionales en hombres y mujeres y sus implicaciones. Revista electrónica de investigación psicoeducativa, 15(6), 455-474.

Shapiro, L. (1997). La inteligencia emocional de los niños. Bilbao: Grupo Zeta.

Soler, S. (2009). Los procesos de reproducción, resistencia y cambio de las relaciones tradicionales de género en la Educación Física: el caso del fútbol. Cultura y educación, 12, 31-42.

Torrents, C., Mateu, M., Planas, A. y Dinusôva, M. (2011). Posibilidades de las tareas de expresión corporal para suscitar emociones en el alumnado. Revista de Psicología del Deporte, 20(2), 401-412. 
Conciencia emocional en situaciones motrices cooperativas lúdicas y expresivas en

Bachillerato: perspectiva de género

José Ignacio Alonso Roque, Marta Marín Guillén, Juan luis Yuste Lucas, Pere Lavega

Burgués y Gemma Gea García

\section{Perfil Académico y profesional de los autores.}

José Ignacio Alonso Roque: Profesor contratado doctor de las asignaturas Educación física en Educación primaria y Juegos motores deportivos en el grado de educación Primaria y de la asignatura de Metodología de la investigación en el Máster de investigación e innovación en Educación infantil y Ed. Primaria de la Universidad de Murcia. Investigación sobre el papel educativo del juego motor e implicaciones emocionales.

Marta Marín Guillén: Licenciada en Ciencias de la Actividad física y del deporte. Máster en Formación de profesorado de la Facultad de Educación de la Universidad de Murcia. Línea de investigación en expresión corporal.

Juan Luis Yuste Lucas: Profesor contratado doctor de las asignaturas Educación física en Educación primaria en el grado de educación Primaria. Profesor de la asignatura de Metodología de la investigación: análisis estadístico, en el Máster de investigación e innovación en Educación infantil y Ed. Primaria de la Universidad de Murcia.

Pere Lavega Burgués: Catedrático del INEFC-Leida y Profesor titular de la asignatura Teoría y práctica del juego. Miembro fundador de la Asociación europea de juegos y Deportes Tradicionales. Investigaciones principales en juegos y deportes tradicionales así como papel pedagógico del juego motor.

Dirección correspondencia autores: Facultad de Educación. Campus de Espinardo, sn, CP. 30100. Universidad de Murcia. Murcia.

E-mail: jialonso@um.es

Tlf: 868888471

Fax: 868884146 